\title{
A Communication Theoretical Analysis of FRET-Based Mobile Ad Hoc Molecular Nanonetworks
}

\author{
Murat Kuscu, Student Member, IEEE and Ozgur B. Akan, Senior Member, IEEE
}

\begin{abstract}
Nanonetworks refer to a group of nano-sized machines with very basic operational capabilities communicating to each other in order to accomplish more complex tasks such as in-body drug delivery, or chemical defense. Realizing reliable and high-rate communication between these nanomachines is a fundamental problem for the practicality of these nanonetworks. Recently, we have proposed a molecular communication method based on Förster Resonance Energy Transfer (FRET) which is a nonradiative excited state energy transfer phenomenon observed among fluorescent molecules, i.e., fluorophores. We have modeled the FRET-based communication channel considering the fluorophores as single-molecular immobile nanomachines, and shown its reliability at high rates, and practicality at the current stage of nanotechnology. In this study, for the first time in the literature, we investigate the network of mobile nanomachines communicating through FRET. We introduce two novel mobile molecular nanonetworks: FRET-based mobile molecular sensor/actor nanonetwork (FRET-MSAN) which is a distributed system of mobile fluorophores acting as sensor or actor node; and FRET-based mobile ad hoc molecular nanonetwork (FRET$M A M N E T$ ) which consists of fluorophore-based nanotransmitter, nanoreceivers and nanorelays. We model the single message propagation based on birth-death processes with continuous time Markov chains. We evaluate the performance of FRETMSAN and FRET-MAMNET in terms of successful transmission probability and mean extinction time of the messages, system throughput, channel capacity and achievable communication rates.
\end{abstract}

Keywords-FRET, nanocommunications, mobile molecular sensor/actor nanonetwork, mobile ad hoc molecular nanonetwork

\section{INTRODUCTION}

$\mathbf{N}$ ANOMACHINES are envisioned as nanoscale-sized machines with basic operation capabilities such as computing, sensing, actuating. The capabilities of these nanomachines can be improved by enabling communication among them. These nanonetworks are envisaged to accomplish more complex tasks ranging from nuclear defense to treatment of many diseases [1]. Many efforts have been devoted to

\footnotetext{
An earlier version of this work was presented at IEEE Med-Hoc-Net'13, Ajaccio, Corsica, France. [18]

The authors are with the Next-generation and Wireless Communications Laboratory (NWCL), Department of Electrical and Electronics Engineering, Koc University, Istanbul, 34450, Turkey (e-mail: mkuscu, akan@ku.edu.tr).

This work was supported in part by the Turkish Scientific and Technical Research Council under grant \#109E257, by the Turkish National Academy of Sciences Distinguished Young Scientist Award Program (TUBA-GEBIP), and by IBM through IBM Faculty Award.
}

develop communication methods for future nanonetworks. Electromagnetic and molecular communications are the most promising approaches [2], [3]. Recently, we have proposed a radically different molecular communication method based on the energy transfer between fluorescent molecules, i.e., fluorophores, which provides high communication rates at the molecular level [4].

Fluorophores, e.g., organic dyes, fluorescent proteins, and quantum dots (QDs), are special molecules that are able to be excited by optical, electrical, chemical or biological energy, and individually relax to the ground state after a random time, i.e., excited-state lifetime, by fluorescing, i.e., releasing a single photon with a wavelength at the range of their emission spectrum [5]. There are many quenching mechanisms that can suppress the fluorescence of the excited-state donor. For example, collision of the excited donor with another molecule can result in quenching of the fluorescence. In case of a collision, the excited energy of the donor is dissipated through heat without radiation of photon. Förster resonance energy transfer (FRET) is another quenching mechanism that defines the migration of the excited-state energy in the form of exciton from the donor molecule to a ground-state acceptor molecule. In the case of FRET, the donor does not fluoresce, and the acceptor non-radiatively becomes excited, i.e., sensitized, by receiving the donor's exciton.

FRET occurs non-radiatively when the donor and acceptor are close to each other with intermolecular distances less than $10 \mathrm{~nm}$ [6]. It is a pairwise energy transfer phenomenon, therefore, an exciton can be transferred to a single acceptor at a time. It is based on the near-field Coulomb interaction of the emission transition dipole moment of the donor with the acceptor's absorption transition dipole moment. Therefore, the phenomenon requires a significant overlap between the donor's emission spectrum and the acceptor's absorption spectrum which increases the resonance probability.

Due to the strong dependence of transfer probability on the intermolecular distance, FRET is extensively exploited as a spectroscopic ruler in biotechnological research [7]. Protein binding events, molecular anisotropy and proximity can be efficiently observed through FRET [8]. There exist several nanoscale biosensors based on FRET mechanism which signal the existence of certain targets in the sensor's proximity [9], [10]. The phenomenon is also naturally observed in photosynthesis during the efficient transmission of absorbed optical energy to the reaction centers [11], thus, FRET constitutes the main focus for the design of synthetic light-harvesting devices 
[12].

FRET has been proposed as a means of short-range nanocommunications for the first time in [4]. The idea was mainly based on encoding the information into the excitedstates of fluorophores, and transferring the information via FRET mechanism between a donor fluorophore as the nanotransmitter antenna and an acceptor fluorophore as the nanoreceiver antenna. In [13], employing a laser with a wavelength in the range of donor's absorption spectrum as the remote information source and using on-off keying (OOK) modulation scheme by representing single bit of information with the presence or absence of a single exciton, we information theoretically analyzed the capacity of the channel between them. We also investigated the pulsed excitation scheme in which the information is encoded into multiple excitons to increase the reliability of the channel [14]. We showed that the high rate of energy transfer between fluorophores $\left(10^{6}-10^{9} \mathrm{~Hz}\right)$ can provide high rate and reliably nanocommunications. Lastly, with a Monte Carlo approach, we simulated the information transmission through point-to-point channel in a three dimensional aqueous medium comprising a varying concentration of relay fluorophores, and showed that information can be reliably transmitted through $200 \mathrm{~nm}$ distance at a rate over $10 \mathrm{Mbps}$ [15]. Due to the high degree of randomness intrinsic to the FRET mechanism, all of these studies assumed that the fluorophores are stationary, and the relative orientation of the fluorophores is constant during the excited-state lifetime, therefore, we applied the classical Förster theory which is not valid for mobile diffusing fluorophores. However, most of the applications that nanonetworks promise, such as inbody target detection and drug delivery, need to employ mobile nanomachines. Therefore, this study aims to investigate the FRET-based communication between mobile nanonodes by approaching the problem from a perspective radically different from classical Förster theory.

In case that the fluorophores with sufficiently long excitedstate lifetimes are used, a donor and acceptor pair with long intermolecular distance at the time of excitation can get into proximity during the lifetime of the donor molecule. Therefore, the probability for an excited donor to get into proximity with more ground-state acceptors during its lifetime increases. As a result of these conditions, the classical Förster theory fails to express the FRET probability for a mobile excited state fluorophore. Fortunately, Stryer et al. derived an expression for the transfer rate of the fluorophores with long lifetimes diffusing in a three dimensional environment [16].

In this study, using the expressions derived in [16], [17], we investigate the performance of FRET-based communication between mobile nanonodes for two network scenarios: i) FRETbased mobile molecular sensor/actor network (FRET-MSAN); and ii) FRET-based mobile ad hoc molecular network (FRET$M A M N E T$ ). In FRET-MSAN, bioluminescent molecules which are special kind of fluorophores that are excited upon binding a target molecule are considered as the sensor nodes. There are also actuators in the network that can realize a specific task upon receiving an exciton. This deployment can be used for autonomous sensing and actuating tasks at the molecular level. FRET-MAMNET consists of transmitter, relay and receiver nanonodes which are also fluorophores. The single nanotransmitter in the network receives a pulsed excitation signal from an information source and sequentially transmits the excitons to the relay nodes or directly to the nanoreceivers in a probabilistic manner.

In order to model the single message propagation in both networks, in the earlier version of this study [18], we exploit the SIR model of epidemics [19] to analytically derive the message transmission probability in FRET-MSAN, and the transmission delay in FRET-MAMNET assuming that a continuous excitation pulse is applied until the detection. However, SIR model is incapable of expressing the transmission probability for FRET-MAMNET with finite-time excitation pulses, and the message transmission delay for FRET-MSAN. In this study, instead of using the SIR models, we benefit from the birthdeath processes with continuous time Markov chain models, and extend our previous study by investigating the transmission delay, system throughput and mutual information for FRET-MSAN. Considering that a finite-time excitation pulse is applied, we analyze the message transmission probability, transmission delay, the information theoretical capacity of the channel between the nanotransmitter and the nanoreceivers, and achievable communication rates for FRET-MAMNET. The performance evaluation is carried out by the Monte Carlo simulations of Markov chain models for both mobile nanonetworks.

The remainder of this paper is organized as follows. In Section II, we review the FRET theory and rapid-diffusion criterion for mobile fluorophores. In Section III, we present the Markov chain models for single message propagation in FRETMSAN and FRET MAMNET. The results of Monte Carlo simulations for performance evaluation are given in Section IV. Finally, the concluding remarks are given in Section V.

\section{THEORY OF FRET}

FRET is a fundamental excited state process observed among fluorescent molecules. It is the transfer of excited state energy in the form of exciton from an excited molecule, called the donor, to a ground-state molecule, called the acceptor, in close proximity of each other. The theory of FRET between immobile fluorophores is established in Theodor Förster's seminal work [6], and then, fundamentally validated by an extensive number of experimental studies [7].

When there exists no quenching mechanism such as molecular collisions or FRET, the excited-state donor is expected to return to ground-state after a randomly short time by fluorescing, i.e., releasing a photon. The excited state lifetime $\tau$, i.e., the time interval between the excitation and relaxation of a molecule, is an exponential random variable of which mean is generally in the range between $2 \mathrm{~ns}-1 \mu \mathrm{s}$ [5]. The reciprocal of the mean excited-state lifetime gives the natural fluorescence rate $k_{0}$ of a fluorophore, i.e., $k_{0}=1 / \mu_{0} . k_{0}$ is the number of photons released at a unit time when there is no quencher. The existence of a quenching mechanism provides additional pathways for the excited-state donor to relax to the ground-state, and thus, significantly reduces the lifetime of excitons by increasing the relaxation rate of the donor.

As a fundamental quenching mechanism, FRET can be realized if the following conditions are satisfied: $i$ ) the excited 
fluorophore must be in close proximity $(0-10 \mathrm{~nm})$ with at least one ground-state acceptor; $i i)$ the emission spectrum of the donor and the absorption spectrum of the acceptor must overlap; and iii) the relative orientation of transition dipole moments of the donor and the acceptor fluorophores must not be orthogonal. If all requirements are met, the rate of the energy transfer as a function of the natural fluorescence rate of the donor $k_{0}$ and in terms of number of excitons transferred per unit time is given as

$$
k_{t}=k_{0}\left(\frac{R_{0}}{R}\right)^{6}
$$

where $R$ is the intermolecular distance, and $R_{0}$ is the Förster radius which incorporates the effects of some intrinsic and environmental parameters. Since information is encoded into the excitons, the exciton transfer rate $k_{t}$ is the most crucial parameter for the performance of FRET-based communications. It is a measure of how fast the information can be transferred between fluorophore-based nanonodes. Förster radius $R_{0}$ can be expressed by

$$
R_{0}=\left(8.8 \times 10^{22} \kappa^{2} n^{-4} Q_{D} J\right)^{-\frac{1}{6}}
$$

where $\kappa^{2}$ is the relative orientation factor, $Q_{D}$ is the quantum yield of the donor, $n$ is the refractive index of the medium, and $J$ is the degree of the spectral overlap. $R_{0}$ ranges between 4 - $10 \mathrm{~nm}$ [5].

In the case of mobile fluorophores, the situation is radically different in the sense that during the excited state lifetime of the donor, the intermolecular distances and the relative orientation of the dipole moments of fluorophores are not constant. Furthermore, the excited donor fluorophore can get in close proximity with a varying number of acceptors if the donor lifetime or the diffusion coefficient of the fluorophores is sufficiently long. Considering that the excitons randomly walk in a random lattice consisting of diffusing fluorophores, giving a closed form expression for the transfer rate requires some assumptions. Stryer et al. postulated the governing rate equations for the energy transfer from a single excited donor to a single ground-state acceptor in a three dimensional environment assuming that the fluorophores are in the rapid-diffusion limit [17]:

$$
k_{r d}=\frac{4 \pi k_{0} R_{0, d-a}^{6}}{3 V a_{d-a}^{-3}}
$$

where $R_{0, d-a}$ is the Förster radius between the donor and the acceptor, and $V$ is the volume of the three dimensional medium. $a_{d-a}$ is the possible closest distance between the centers of the donor and the acceptor. When there are more than one acceptor molecules, the total FRET rate between the donor and the acceptors becomes

$$
k_{t}=k_{r d} N_{a}
$$

where $N_{a}$ is the number of available, i.e., ground-state, acceptor molecules in the environment. The rapid-diffusion criterion is given as

$$
\frac{D \tau_{0}}{s^{2}} \gg 1
$$

where $D$ is the sum of diffusion coefficients of the donor and the acceptor, $\tau_{0}$ is the natural excited state lifetime of the donor, i.e., $\tau_{0}=1 / k_{0}$, and $s$ is the mean intermolecular distance between donor-acceptor fluorophores [17], [20].

The rapid diffusion limit can be achieved by using fluorophores with moderate diffusion coefficients and long lifetimes such as $1-2 \mu s$ [20].

\section{FRET-BASED MOBILE MOLECUlAR NANONETWORKS}

FRET-based nanocommunication channel has been previously modeled with different configurations assuming that the communicating nanomachines are immobile during the communication. However, most of the applications, especially in-body applications, that nanonetworks promise, require for nanomachines to be mobile. Along with this motivation, in this section, we focus on the network of mobile nanomachines communicating through FRET. We model the single message propagation both in FRET-MSAN and FRET-MAMNET based on Continuous Time Markov Chains (CTMCs) assuming that the nanonetwork nodes are freely and randomly diffusing in a three dimensional aqueous environment satisfying the rapiddiffusion criterion.

The performance of the proposed nanonetworks are investigated based on the results of the Monte Carlo simulations on Markov chain models. For FRET-MSAN, we obtain the successful transmission probability of a single message generated on the nanosensors to the nanoactors randomly diffusing in the environment. The temporal characteristics of the network are analyzed based on the average delay between the generation of a single message on the nanosensors and its reception by the nanoactors. We derive the average system throughput using the delay parameter and the number of nanoactors that receive the message. We also information theoretically investigate the mutual information between nanosensors and nanoactors with varying event probability. For FRET-MAMNET, the information theoretical capacity of the communication channel between the nanotransmitter and nanoreceivers is obtained through the successful transmission probability of a single message for varying system parameters. We also investigate the achievable communication rate on the mobile network based on the transmission delay.

\section{A. FRET-Based Mobile Molecular Sensor/Actor Network (FRET-MSAN)}

Bioluminescent molecules define a class of fluorescent molecules which are excited upon binding a target molecule [5]. Since they do not need a remote excitation source, e.g., optical laser, they are extensively used in biotechnological research as biomolecular sensors optically indicating the presence of a certain kind of molecule [21]. For example, aequorin, a bioluminescent protein, reacts with calcium ions, and relaxes through releasing a photon, thus, it is extensively used to measure $\mathrm{Ca} 2+$ concentration [22].

Fluorescent molecules also find applications in photodynamic therapy (PDT) of cancer as actuators. In QD-based PDT, QDs are excited by optical energy from a remote source 


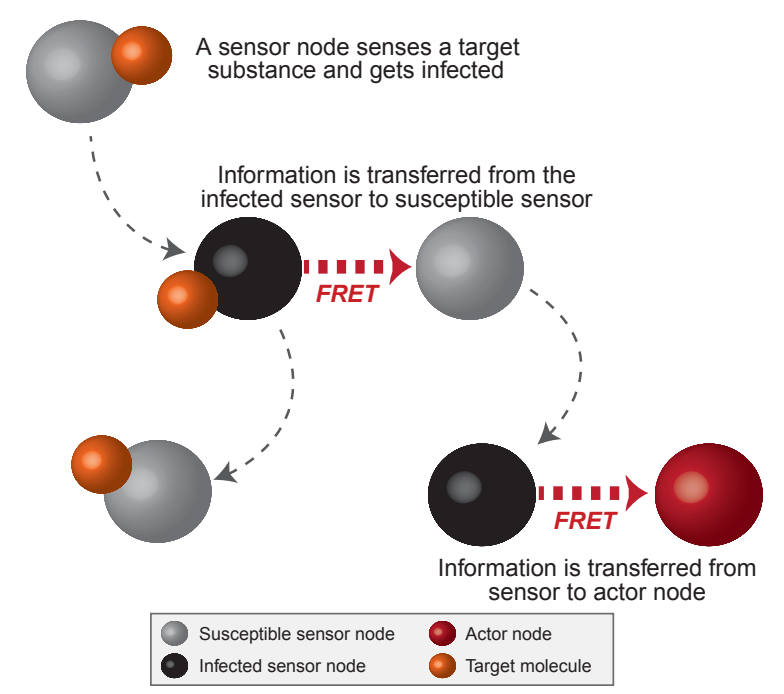

Fig. 1. Information flow in FRET-MSAN.

and then transfer its exciton to the conjugated photosensitizing agent which synthesizes a reactive singlet oxygen via energy transfer [23]. The produced singlet oxygen initiates the apoptosis of nearby cancer cells. However, the reactive singlet oxygen is also harmful for normal cells, therefore, the spatial precision of the activation of singlet oxygen is crucial.

Here, we focus on a molecular sensor and actor network, namely FRET-MSAN, composed of mobile bioluminescent sensors and fluorophore-based actors that can collect the information from the sensors and perform an appropriate action upon the environment. The investigated scenario in this section can pave the way for designing autonomous networks of nanomachines which are able to collaboratively sense the presence of tumor cells, and act precisely for the apoptosis of them. The information flow in FRET-MSAN is demonstrated in Fig. 1.

The generated message by the nanosensors is a one-bit message which is an exciton indicating whether the nanosensors bound to a target molecule or not. The target can be a macromolecule, e.g., biopolymer, and thus, it is possible that more than one sensor nodes bind to the same target. In this case, each nanosensor can generate excitons signaling the existence of the target, therefore, more than one excitons can flow on the network at the same time. We assume that the nanoactors are not capable of the detecting the target and generating excitons individually. The initially generated excitons occupy on the detecting nanosensors during an exponentially random time, and then transferred to the other sensors or actors in the ground-state, i.e., randomly walk on a random lattice, or they can be removed from the system by fluorescence.

We assume that the detection is realized with a varying number of nanosensor nodes at the same, and during the transmission of the detection message, no other detection occurs. Therefore, the initially generated excitons set an upper bound on the number of excitons on the network, and the number of excitons is a monotonically decreasing function.

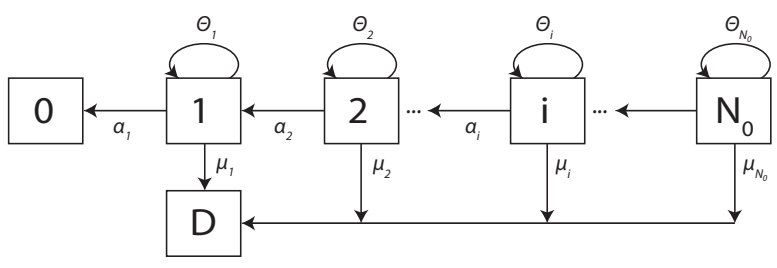

Fig. 2. Markov chain model of FRET-MSAN.

During this discrete fade away of the excitons, if at least one nanoactor receive an exciton from an excited sensor node, the message is successfully transferred. These characteristics of the network imply that the single message transmission resembles a death process which can be modeled with a continuous time Markov chain demonstrated in Fig. 2.

In the model, the states demonstrate the number of excitons that are available on the network. The transition rates depend on the state of the network, i.e., number of excitons that randomly walk throughout the network. There are two absorbing states in this Markov chain, i.e., state-0 and state-D. The state- 0 represents the complete extinction of the excitons in the network. Detection cannot be realized once the extinction occurs since no other exciton is generated. If none of the nanoactors receive an exciton until the extinction, the message is lost, and the transmission fails. The state-D represents the successful detection of the message which occurs when a nanoactor receives an exciton. All of the states except the state- 0 are connected to the state-D. The model is based on the following assumptions:

- Initially $N_{0}$ number of sensors are in the excited state, i.e., $N_{0}$ number of sensors detect the target.

- No additional exciton is generated during the message propagation.

- The number of sensor nodes, $N_{s}$, and the number of actor nodes, $N_{a}$, and the total number of the network nodes, $N=N_{s}+N_{a}$, in the closed environment are constant.

- An excited sensor node gets rid of the excitation and returns to the ground-state without an exciton transfer at a rate of $k_{0}$. Note that, this is the natural fluorescence rate of the bioluminescent fluorophores used as molecular sensors.

- An excited sensor node transfers the infection to a ground-state sensor node making it excited while returning to the ground-state with a rate of $k_{s s}$.

- An excited sensor node transfers its excitation to an actor, and return to the ground-state with a rate of $k_{s a}$.

- The exciton transfer is pairwise, i.e., an excited nanonode can transfer its excitation to only a single nanonode at a time.

The information transfer rate between a rapidly-moving excited sensor node to a ground-state, i.e., available, sensor node in the environment, i.e., $k_{s s}$, is given using (3) as follows

$$
k_{s s}=\frac{4 \pi k_{0} R_{0, s s}^{6}}{3 V a_{s s}^{-3}}
$$


where $R_{0, s s}$ is the Förster radius between sensor nodes, and $a_{s s}$ is the intermolecular distance of closest approach of two sensor nodes. We assume that the bioluminescent sensors are spherical with radius $r_{s}$, therefore, $a_{s s}=2 r_{s}$. Similarly, the rate of the information transfer from an excited sensor node to an actor node is given by

$$
k_{s a}=\frac{4 \pi k_{0} R_{0, s a}^{6}}{3 V a_{s a}^{-3}}
$$

where $R_{0, s a}$ is the Förster radius between a sensor node and an actor node, and $a_{s a}$ is the intermolecular distance of closest approach of a sensor node with an actor node. Assuming that the actor nodes are also spherical with radius $r_{a}, a_{s a}=r_{s}+r_{a}$.

Based on the listed assumptions, and using the transfer rates in rapid-diffusion, we derive the transition rates of the Markov chain. The state-dependent death rate, $\alpha_{i}$, is the overall fluorescence rate on the network, and it can be given by

$$
\alpha_{i}=i k_{0}
$$

where $i$ is the number of excitons, i.e., number of excited sensor nodes, on the network, and $k_{0}$ is the natural fluorescence rate of the fluorophores which we assume identical for each fluorophore. The exciton transfers between sensor nodes do not alter the state of the network, since the number of excitons does not change. The overall transfer rate between nanosensors is given in terms of number of excited state sensors and groundstate sensors as

$$
\theta_{i}=i\left(N_{s}-i\right) k_{s s}
$$

where $N_{s}$ is the total number of sensor nodes. The rate of transfer from the excited sensor nodes to the actor nodes can be given by

$$
\mu_{i}=i N_{a} k_{s a}
$$

where $N_{a}$ is the number of actor nodes.

Considering the infeasibility of deriving analytical expressions for the performance metrics for this death process, we simulate the model by dividing the time into small intervals, i.e., $\Delta t$, in which we assume only one event, e.g., exciton transfer or fluorescence, can occur. For this time interval, the transition probabilities can be given as

$$
\begin{gathered}
P_{i, i-1}=P\left(S_{t+\Delta t}=i-1 \mid S_{t}=i\right) \approx \alpha_{i} \Delta t \\
P_{i, i+1}=P\left(S_{t+\Delta t}=i+1 \mid S_{t}=i\right)=0 \\
P_{i, D}=P\left(S_{t+\Delta t}=D \mid S_{t}=i\right) \approx \mu_{i} \Delta t \\
P\left(S_{t+\Delta t}=i \mid S_{t}=i\right) \approx 1-P_{i, i-1}-P_{i, D}
\end{gathered}
$$

where $i=1,2, \ldots N_{0}$, and $S_{t}$ demonstrates the state of the network at time $t$. Using the transition probabilities and setting a sufficiently small time interval $\Delta t$, we conduct a Monte Carlo simulation in Matlab to obtain the successful detection probability of a single message, $\operatorname{Pr}$ (success) and average message delivery delay, $E\left[T_{d}\right]$ for different system parameters. The detection probability is calculated as the number of successful transmission to the number of extinctions without detection. The simulation is run until the detection probability converges to a finite value. In the simulations, we also obtain the average number of actor nodes that receive an exciton for each cycle of message transmission, i.e., $n$. Using this parameter and the mean delay as the average extinction time of the excitons, we derive the average system throughput as

$$
T_{\text {avg }}=\frac{n}{E\left[T_{d}\right]}
$$

If there is sufficient interval between each target detection, intersymbol interference (ISI) does not occur. Since we assume that no other excitons except the initial excitons are generated during the transmission, no-target message, i.e., bit-0, is always successfully detected if there is no-ISI. Considering that only the transmission of bit-1 is problematic, the communication channel between nanosensors and nanoactors is information theoretically a Z-channel. Therefore, considering $X$ as the transmitted bit by the sensor nodes, and $Y$ as the received bit by the actor nodes, the mutual information between $X$ and $Y$ can be expressed as

$I(X ; Y)=H\left(P_{E} \operatorname{Pr}(\right.$ Success $\left.)\right)-P_{E} H(1-\operatorname{Pr}($ Success $))$,

where $H($.$) is the binary entropy function, and P_{E}$ is the event probability.

The derived metrics based on the simulations are sufficient to evaluate the performance of the FRET-MSAN with different system parameters. The results of the simulation and the network performance based on the varying parameters are discussed in the Section IV.

\section{B. FRET-Based Mobile Ad Hoc Molecular Network (FRET- $M A M N E T$ )}

In FRET-MAMNET, there are three kinds of fluorophorebased nanonodes randomly dispersed in the same environment: i) nanotransmitter; ii) nanorelays; and iii) nanoreceivers. The single nanotransmitter in the network is continuously excited by an information source, e.g., a remote optical source or a nearby electrical source, with a pulse of duration $T_{p}$. The excited transmitter node generates excitons randomly during $T_{p}$. We can assume that the nanotransmitter is always occupied by an exciton, therefore, it is always in the excited-state. The relay nodes are not directly excited by an information source. The excitons generated on the nanotransmitter follow random hopping with sequential transfers through the relay nodes, and carry the message from the nanotransmitter to the nanoreceivers. The nanoreceivers are assumed to realize a specific task, e.g., singlet oxygen sensitization, when they receive an exciton. This configuration is similar to the scenario simulated in [15], however, here we assume that the characteristics of the network nodes satisfy the rapid-diffusion limit, therefore, we are able to derive closed form expression for transfer rates among fluorophores. The information flow in FRET-MAMNET is demonstrated in Fig. 3.

We model the single message propagation from the nanotransmitter to a nanoreceiver until an exciton is first received by an receiver node. Different from the FRET-MSAN, in this network excitons are generated by the information source. Therefore, the transmission of a single message during the application of the excitation pulse is a birth-death process 


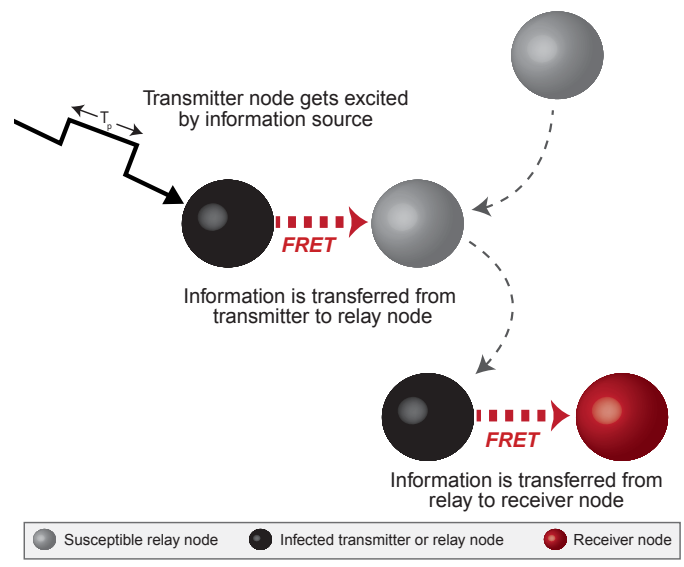

Fig. 3. Information flow in FRET-MAMNET.

which can be modeled by the Markov chains demonstrated in Fig. 4(a). The upper limit for the number of excitons in this model can be given as the total number of relay and transmitter nodes, i.e., $N_{h}$. The Markov chain in Fig. 4(b) models the message propagation for the time from the cut-off of excitation pulse until the complete extinction of the excitons. This second model describing the no-pulse situation is similar to the Markov chain model of FRET-MSAN, where no exciton generation occurs. The upper limit for the second model is the number of excitons that exists on the network at the time of pulse cut-off, i.e., $N_{p}$. Note that, the message can be detected during the excitation pulse, i.e., before the second model is applied. However, the network never goes into state-0 during the pulse, since the nanotransmitter is always excited by the excitation pulse.

The model is based on the following assumptions:

- Transmitter and relay nodes are of same type of fluorophores, i.e., they have the same fluorescence rate $\left(k_{0}\right)$, Förster radius $\left(R_{0, h h}\right)$ and molecular radius $\left(r_{h}\right)$.

- There is only a single nanotransmitter that can receive excitation signal from the information source.

- An excited nanonode gets rid of the excitation and returns to the ground-state without transfer of exciton at a rate of $k_{0}$.

- An excited nanonode transfers the excitation to a groundstate nanonode with a rate of $k_{h h}$.

- An excited nanonode transfers the excitation to a nanoreceiver with a rate of $k_{h r}$.

- During the pulse, excitons are generated on the transmitter node with a rate of $k_{g}$.

- The total number of transmitter and relay nodes, $N_{h}$, and the number of receiver nodes, $N_{r}$, are constant.

On the nanotransmitter, when an exciton is removed either by self relaxation, i.e., fluorescence, or by transfer to another node, another exciton is generated immediately during the application of the excitation pulse. Therefore, the exciton generation rate on the nanotransmitter is given by

$$
k_{g}(t)=k_{0}+k_{h h}\left(N_{h}-N_{h, e}(t)\right)
$$

(a)

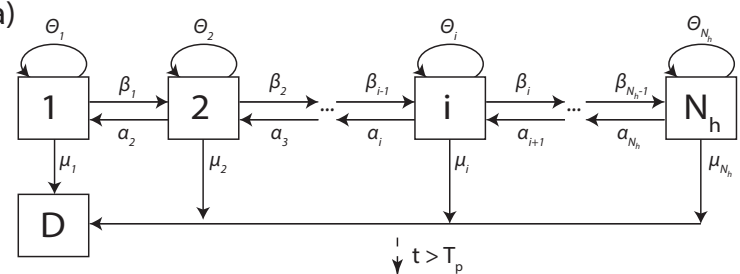

(b)

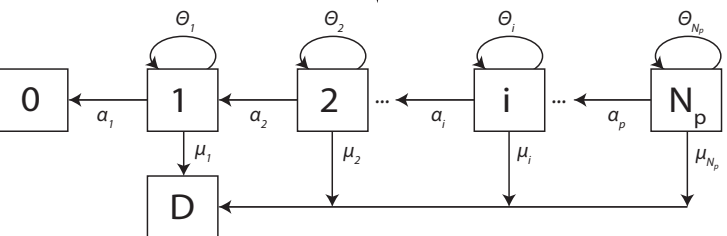

Fig. 4. Markov chain model of FRET-MAMNET.

where $N_{h, e}(t)$ is the number of excited nanotransmitters and nanorelays at time $t$. $k_{h h}$ is the transfer rate between a single transmitter-relay (or relay-relay) pair, that can be expressed using (3) as

$$
k_{h h}=\frac{4 \pi k_{0} R_{0, h h}^{6}}{3 V a_{h h}^{-3}}
$$

where $R_{h h}$ is the Förster radius between transmitter-relay (or relay-relay) pair, and $a_{h h}$ is the intermolecular distance at the closest approach of a relay node with a relay or transmitter node. In a similar way, the exciton transfer rate between an excited relay (or transmitter) and a receiver node is given as

$$
k_{h r}=\frac{4 \pi k_{0} R_{0, h r}^{6}}{3 V a_{h r}^{-3}}
$$

where $R_{h r}$ is the Förster radius between a nanoreceiver and a nanotransmitter or nanorelay, and $a_{h r}$ is the intermolecular distance at the closest approach of a nanoreceiver with a nanorelay or nanotransmitter.

The state-dependent transition rates on the Markov chain can be expressed in terms of the exciton transfer, generation, and fluorescence rates as follows

$$
\begin{gathered}
\alpha_{i}=i k_{0} \\
\theta_{i}=i\left(N_{h}-i\right) k_{h h} \\
\mu_{i}=i N_{r} k_{h r}
\end{gathered}
$$

where $i$ always denotes the number of excitons on the network. The birth rate during the excitation pulse can be expressed as the generation rate of the excitons, i.e,

$$
\beta_{i}=k_{g, i}=k_{0}+k_{h h}\left(N_{h}-i\right)
$$

Assuming again that the time is divided by sufficiently small intervals, $\Delta t$, the transition probabilities can be expressed as follows

$$
\begin{aligned}
& P_{i, i-1}=P\left(S_{t+\Delta t}=i-1 \mid S_{t}=i\right) \approx \alpha_{i} \Delta t \\
& P_{i, i+1}=P\left(S_{t+\Delta t}=i+1 \mid S_{t}=i\right) \approx \beta_{i} \Delta t
\end{aligned}
$$




$$
\begin{gathered}
P_{i, D}=P\left(S_{t+\Delta t}=D \mid S_{t}=i\right) \approx \mu_{i} \Delta t \\
P\left(S_{t+\Delta t}=i \mid S_{t}=i\right) \approx 1-P_{i, i-1}-P_{i, i+1}-P_{i, D}
\end{gathered}
$$

where $i=1,2, \ldots N_{h}$.

Using the transition probabilities for the time interval $\Delta t$, we again conduct Monte Carlo simulation to obtain the successful detection probability of the information generated on nanotransmitter, and the mean extinction time of the excitons which we name as the mean delay, i.e., $E\left[T_{d}\right]$. Based on the obtained transmission probabilities, we also investigate the information theoretical capacity of the channel between nanotransmitter and the nanoreceivers. Assuming a sufficient time interval between successive generation of messages on the nanotransmitter, we can neglect the ISI. Therefore, the channel shows Z-channel characteristics as in the FRET-MSAN, and its capacity can be expressed as the maximum mutual information over all input distributions, i.e.,

$$
C=\max _{P_{1}} I(\mathbf{X} ; \mathbf{Y})
$$

where $P_{1}$ is the probability that the information source generates bit-1. Since this time we have control over the information source different from the case of FRET-MSAN where targets can be detected randomly, the capacity can be achieved. We derive the achievable transmission rates for the channel between nanotransmitter and nanoreceivers as follows,

$$
R=\frac{C}{E\left[T_{d}\right]}
$$

assuming that a bit interval of $E\left[T_{d}\right]$, i.e., mean extinction time of excitons, is sufficient to neglect ISI.

\section{Performance Analysis of FRET-Based Mobile MOLECULAR NANONETWORKS}

In this section, we evaluate the performance of the two network scenario described in Section III. We conduct MonteCarlo simulations to obtain the successful detection probability of a single message and the mean extinction time of excitons in FRET-MSAN and FRET-MAMNET with varying network and node parameters to understand the effect of each parameter on the network performance and gain insight on the feasibility of the FRET-based mobile nanonetworks.

Note that the Markov chain models developed in the previous section are not adequate to reveal the spatial properties of these mobile nanonetworks such as communication coverage, since they are based on the number of excitons on the whole network regardless of their location assuming that the rapiddiffusion criterion is satisfied. The spatial properties of information flow can be observed by computationally expensive algorithms that do not necessitate rapid diffusion and incorporate the random Brownian motion of network nodes. This is left as a future work. Nevertheless, we know from the work in [15] that when the network nodes are assumed to be immobile the information can be transferred over a range of $200 \mathrm{~nm}$.
TABLE I. SimUlation Parameters FOR FRET-MSAN

\begin{tabular}{l|l}
\hline \hline Radius of nanosensors $\left(r_{s}\right)$ & $0.25(\mathrm{~nm})$ \\
\hline Radius of nanoactors $\left(r_{a}\right)$ & $0.25(\mathrm{~nm})$ \\
\hline $\begin{array}{l}\text { Natural fluorescence rate of sensor } \\
\text { and actor nodes }\left(k_{0}\right)\end{array}$ & $10^{6}\left(\mathrm{sec}^{-1}\right)$ \\
\hline Volume $(V)$ & $1\left(\mu \mathrm{m}^{3}\right)$ \\
\hline $\begin{array}{l}\text { Number of initially excited sensor } \\
\text { nodes }\left(N_{0}\right)\end{array}$ & 5 \\
\hline Total number of sensor nodes $\left(N_{s}\right)$ & 100 \\
\hline Number of actor nodes $\left(N_{a}\right)$ & 20 \\
\hline $\begin{array}{l}\text { Förster radius of nanosensor- } \\
\text { nanosensor pair }\left(R_{0, s s}\right)\end{array}$ & $8(\mathrm{~nm})$ \\
\hline $\begin{array}{l}\text { Förster radius of nanosensor- } \\
\text { nanoactor pair }\left(R_{0, s a}\right)\end{array}$ & $8(\mathrm{~nm})$ \\
\hline
\end{tabular}

\section{A. Performance Analysis of FRET-MSAN}

In this section, we present the performance of the FRETMSAN based on the simulation results for single message propagation. We analyze the single-message transmission probability, mean transmission delay as the mean extinction time of excitons, average system throughput and mutual information between the generated and the detected message for varying number of nanoactors and initially excited nanosensors, varying Förster radius between nanosensors and nanoactor, and varying size of nanomachines. Note that, the number of initially ground-state nanosensors, the Förster radius of nanosensor-nanosensor pair, and the fluorescence rate of the nanonodes do not affect the detection probability. The default values of parameters used in the numerical simulations are presented in Table I.

1) Probability of Successful Detection: The effect of the number of initially infected nanosensors on the detection probability is shown in Fig. 5(b) with varying number of nanoreceivers. Since we assume that no other external infection occurs, and the infection is only transferred between the network nodes, the number of infected nanosensors is a decreasing function of time. Therefore, the initial number of infection is an important parameter for the detection performance. It is observed that the detection probability significantly increases even with a small increase in the number of initially infected nanosensors.

The probability of detection with varying number of nanoactors, $N_{a}$, for different values of Förster radius of nanosensornanoactor pair, $R_{0, s a}$, is shown in Fig. 5(a). We observe that the detection probability significantly increases with increasing number of actors, since the chance for an infected nanosensor to be in the communication range of an actor node at any time increases.

In Fig. 5(c), $\operatorname{Pr}$ (success) with varying $R_{0, s a}$ for different number of receiver nodes is presented. It is shown that as $R_{0, s a}$ increases, the probability of detection is significantly enhanced. In fact, Förster radius is analogous of the communication range of a network node in traditional networks. Therefore, with a large communication range of a nanosensor, more nanoactors as acceptors become available for the energy transfer. As a result, the transfer probability of excitons on each node 


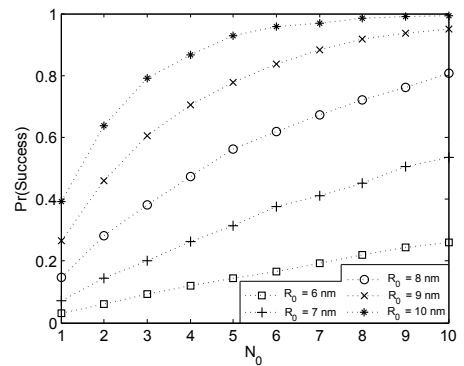

(a) $\operatorname{Pr}$ (success) with varying $N_{0}$ for different $R_{0, s s}=R_{0, s a}=R_{0}$.

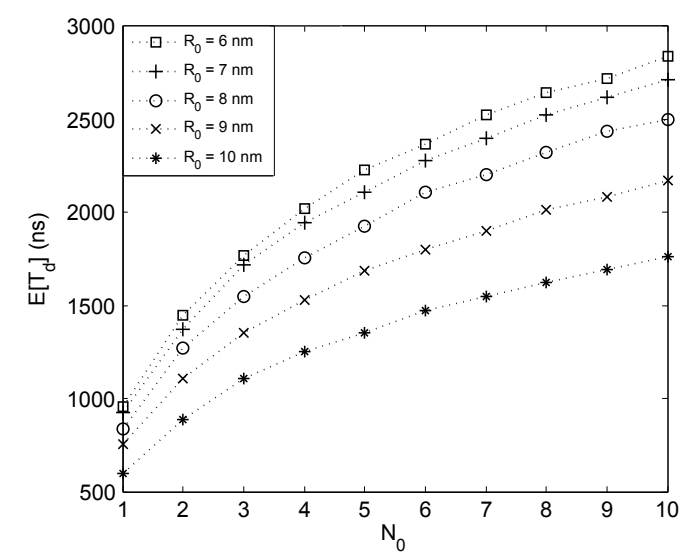

(d) $E\left(T_{d}\right)$ with varying $N_{0}$ for different $R_{0}$ $R_{0}$.

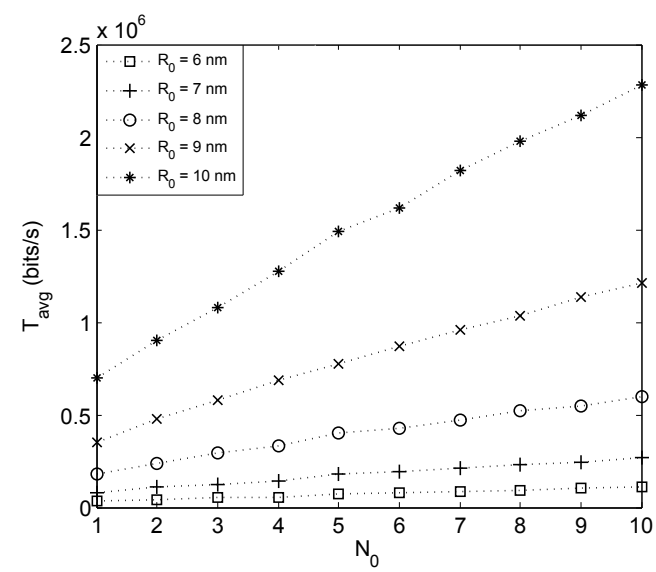

(f) $T_{a v g}$ with varying $N_{0}$ for different $R_{0, s s}=R_{0, s a}=($ $R_{0}$

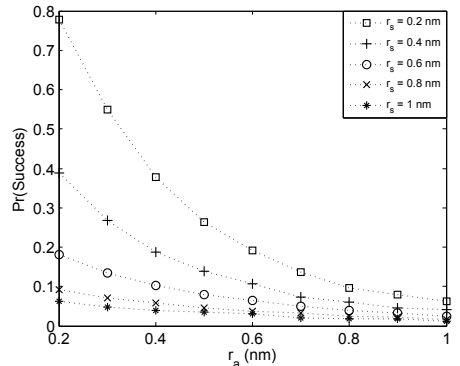

(c) $\operatorname{Pr}$ (success) with varying $r_{a}$ for different $r_{s}$.

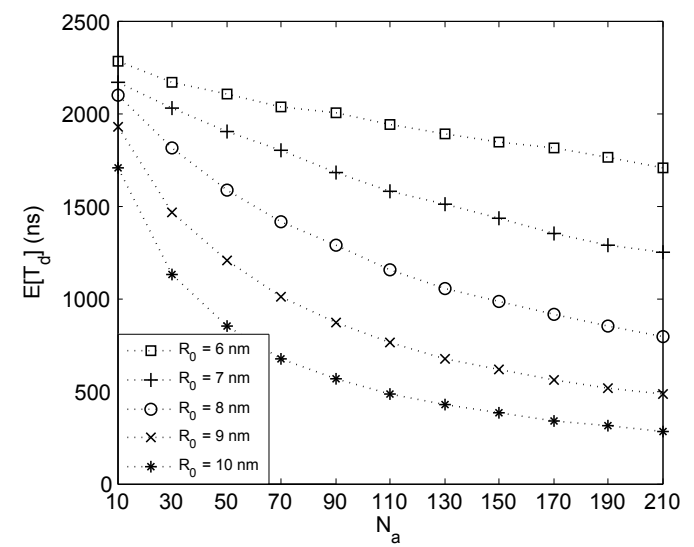

(e) $E\left(T_{d}\right)$ with varying $N_{a}$ for different $R_{0, s s}=R_{0, s a}=$ $R_{0}$.

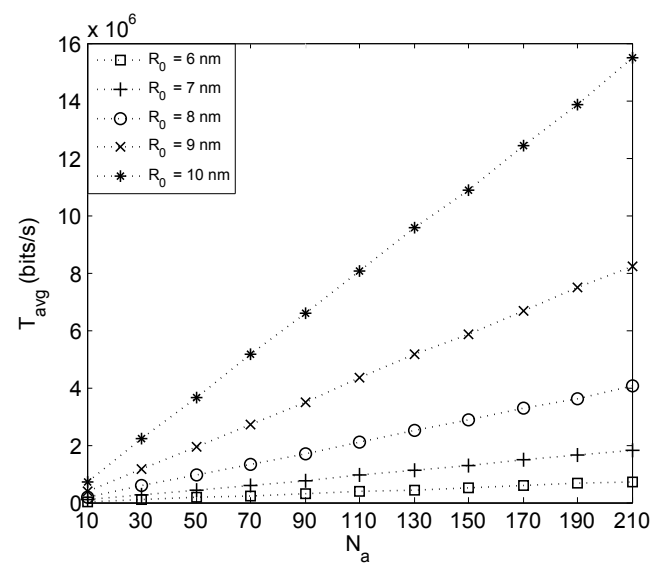

(g) $T_{a v g}$ with varying $N_{a}$ for different $R_{0, s s}=R_{0, s a}=$ $R_{0}$.

Fig. 5. The successful detection probability, mean extinction time of excitons, and average system throughput for one-bit message transmission in FRET-MSAN.

increases with a decrease in the removal probability of excitons by fluorescence. We also observe that for small values of $R_{0, s i}$, a large number of nanoreceivers are required to successfully detect the message of nanosensors. However, by employing nanosensor-nanoactor pairs with relatively large $R_{0, s a}$, less than 50 receiver nodes will be enough for successful detection.

The size of nanomachines also significantly affects the successful detection probability, since the extent of the distance of closest approach has a direct effect on the energy transfer rate between nanonodes. The detection probability for varying radii of nanosensors and nanoactors is shown in Fig. 5(d). The selected values for radii are in the range of size of common fluorophores, e.g., fluorescent dyes. We observe that, using small-size nodes significantly increases the detection probability. 
2) Mean Extinction Time of Excitons: Mean extinction time of the exciton determine the average time that the generated message (bit-1) propagates through the network. Since the actor nodes can get the excitation even when there is a single exciton existing in the network, the mean extinction time, $E\left[T_{d}\right]$ can be regarded as the mean delay for the message transmission, therefore it is one of the main characteristics of the mobile network.

In Fig. 5(d), the mean extinction time is demonstrated with varying $N_{0}$ for different Förster radii. As expected, when the message is encoded into higher number of initial excitons, the extinction time increases, since the initial state of the network is further from the extinction state, i.e., state-0, when there are more initial excitons.

Förster radius between sensor nodes, $R_{0, s s}$, has no effect on the average decay time of the excitons, since it only has a contribution in the rate of the exciton transfer between sensor nodes which does not alter the state of network, i.e., the number of excitons. However, Förster radius between sensor and actor nodes, $R_{0, s a}$ has a significant effect on the extinction time. This is because, increasing $R_{0, s a}$ increases the exciton transfer rate from sensor nodes to actor nodes, i.e., $k_{s a}$. Since an exciton that is transferred to an actor node is assumed to be lost during the decoding process, the number of excitons decreases more rapidly, when $k_{s a}$ increases. This is directly related to the result presented in Fig. 5(e) which demonstrates that the mean extinction time decreases with increasing number of actor nodes which also increases $k_{s a}$.

3) System Throughput: System throughput is a significant factor which determines how many actor nodes can receive the one-bit message per unit time. To calculate the system throughput, the number of actor nodes that achieve to receive excitation until the extinction of the excitons, is observed in the Monte Carlo simulations. The average system throughput that is calculated using (15) is demonstrated in Fig. 5(f) and Fig. 5(g) with varying $N_{0}, N_{a}$ and Förster radii.

When the initial number of excitons increases, the extinction time of the excitons also increases, therefore it is more likely that more actor nodes get into close proximity of excited sensor nodes and get an excitation. Increasing the number of actor nodes and the Förster radius between sensor and actor nodes, the exciton transfer rate from excited sensor nodes to the actor nodes increases. This results in a higher detection probability at any time interval. Therefore, excitons can be transferred to more actor nodes during a single message transmission.

4) Mutual Information: Mutual information between the transmitted message and the detected message is a measure of the reliability of the communication. It is a function of the probability distribution of the transmitted message. In FRETMSAN, the input message is the target detection signal which is encoded into excitons. We refer to detection message as bit-1. Bit- 0 denotes the no-target information, and assuming that no other excitation except the initial ones occurs during the transmission, the correct transmission probability of bit- 0 is always 1 . However, the probability of the correct transmission of bit-1, i.e., target information, depends on the system parameters.

Here, we investigate the mutual information between the transmitted and received alphabets for varying number of initial excitons and actor nodes, and for different molecular sizes of nanosensors and nanoactors. When the number of initial excitons and actor nodes increase and the size of the nanonodes is reduced, the successful transmission probability of bit-1 significantly increases. As a result the mutual information also increases as is seen in Fig. 6.

\section{B. Performance Analysis of FRET-MAMNET}

In this section, we present the results of numerical simulations to investigate the performance of FRET-MAMNET in terms of probability of successful transmission of bit-1, mean extinction time of excitons, information theoretical channel capacity and achievable communication rates. We evaluate the effect of varying number network nodes, varying Förster radii of different pairs, varying lifetime of the nanosensors, varying size of nanonodes, and varying pulse length on these performance metrics. Note that, different from FRET-MSAN, the number of ground-state nanosensors and the excited state lifetime of nanosensors have effect on the performance of FRET-MAMNET through their effect on the generation rate of excitons. The default values of the system parameters used in the simulations are given in Table II.

TABLE II. SimUlation PARAMETERS FOR FRET-MAMNET

\begin{tabular}{l|l}
\hline \hline $\begin{array}{l}\text { Radius of nanotransmitters and } \\
\text { nanorelays }\left(r_{h}\right)\end{array}$ & $0.25(\mathrm{~nm})$ \\
\hline Radius of nanoreceivers $\left(r_{r}\right)$ & $0.25(\mathrm{~nm})$ \\
\hline Excitation pulse length $\left(T_{p}\right)$ & $2(\mu \mathrm{s})$ \\
\hline $\begin{array}{l}\text { Natural fluorescence rate of nano- } \\
\text { transmitter, nanorelay and nano- } \\
\text { receivers }\left(k_{0}\right)\end{array}$ & $10^{6}\left(\mathrm{sec}^{-1}\right)$ \\
\hline Volume $(V)$ & $1\left(\mu \mathrm{m}^{3}\right)$ \\
\hline $\begin{array}{l}\text { Total number of transmitter and } \\
\text { relay nodes }\left(N_{h}\right)\end{array}$ & 100 \\
\hline Number of receiver nodes $\left(N_{r}\right)$ & 20 \\
\hline $\begin{array}{l}\text { Förster radii of nanotransmitter- } \\
\text { nanorelay and nanorelay-nanorelay } \\
\text { pairs }\left(R_{0, h h}\right)\end{array}$ & $8(\mathrm{~nm})$ \\
\hline $\begin{array}{l}\text { Förster radii of nanorelay- } \\
\text { nanoreceiver and nanotransmitter- } \\
\text { nanoreceiver pairs }\left(R_{0, h r}\right)\end{array}$ & $8(\mathrm{~nm})$ \\
\hline
\end{tabular}

1) Probability of Successful Detection of Bit-1: Here, we investigate the effect of pulse length, mean excited state lifetime of the fluorophores, the number of relay nodes, and the Förster radii between fluorophores on the detection probability of bit-1.

The result presented in Fig. 7(a) reveals that the pulse length has a significant effect on the success probability. This is because it determines the number of transmitted excitons into the network. As the pulse length increases, the excitons stay longer time on the network which increases the probability for a receiver node to get into the range of an excited relay or transmitter node. As a result, the receivers can detect the message with a higher probability. The increasing Förster 


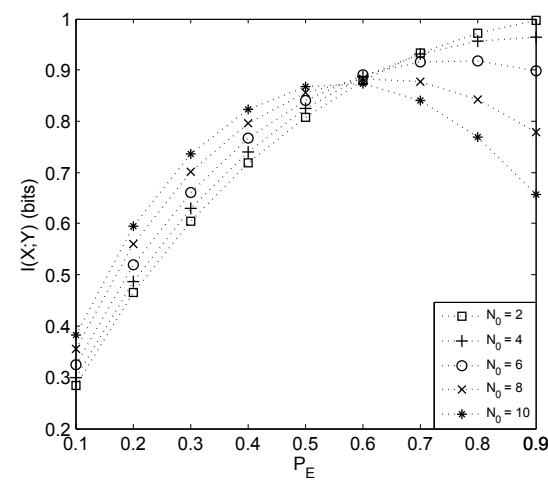

(a) $I(X ; Y)$ with varying $P_{E}$ for different $N_{0}$.

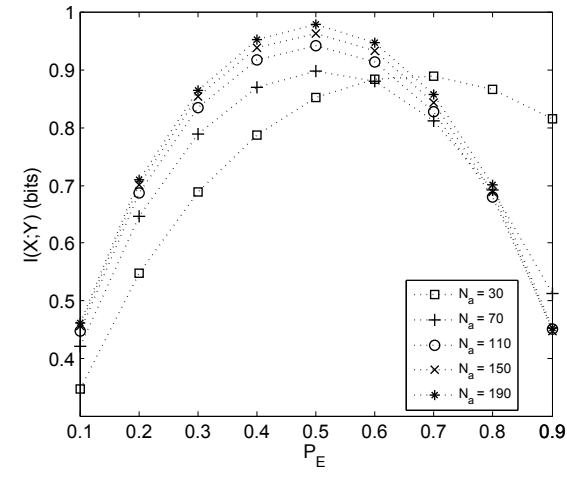

(b) $I(X ; Y)$ with varying $P_{E}$ for different $N_{a}$.

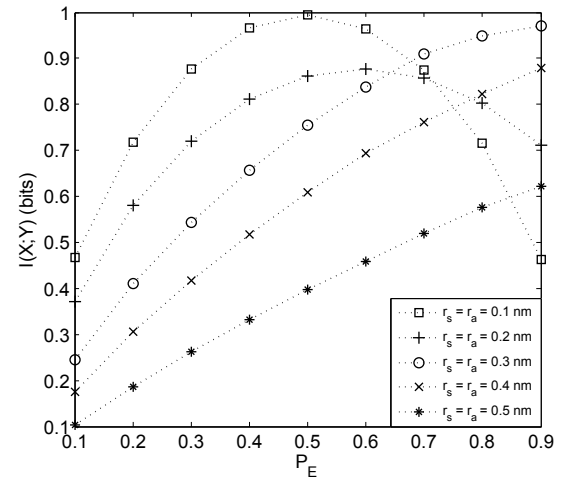

(c) $I(X ; Y)$ with varying $P_{E}$ for different $r_{s}, r_{a}$.

Fig. 6. Mutual information between the generated message on nanosensors and detected message by the nanoactors in FRET-MSAN.

radius between the relay and transmitter fluorophores, $R_{0, h h}$ increases the transfer rate from the nanotransmitter to the nanorelays, therefore, excitons are generated more rapidly on the nanotransmitter. This results in a higher number of excitons existing on the network at the same time which increases the detection probability. When the Förster radius between relay and receiver nodes $R_{0, h r}$ increases, the transfer probability of the excitons from the excited relay nodes to the receiver nodes increases, i.e., detection probability increases.

The effect of excited state lifetime of the nanonodes, $\tau_{0}$, is shown in Fig. 7(b) for different $N_{h}$. It is shown that increasing the lifetime decreases the detection probability. The lifetime has a significant effect on the transmitter side, such that, it directly affects the exciton generation rate. Lifetime gives a measure of average occupation time of an exciton on a nanonode. When the lifetime is short, the excitons are generated more frequently on the nanotransmitter, therefore, the number of excited nanonodes on the network at time $t$ increases, as a result the detection probability increases. It is also clearly shown that the detection probability significantly increases with increasing number of nanorelays. Increasing the concentration of the relay nodes increases the number of ground-state nodes in the range of an excited relay node, thus, increases the transfer rate for each of the nanonode, and excitons stay longer time in the network. Keeping the number of excitons high increases the probability for a receiver to encounter an excited nanonode in its range, and thus increases the detection probability of bit-1.

2) Mean Extinction Time of Excitons: Mean extinction time is investigated for varying pulse length and varying excited state lifetime of the fluorophores. Fig. 7(c) reveals that the mean extinction time almost linearly increases with the increasing pulse length. This is expected because the excitation pulse prevents the network from entering into the extinction state, such that, the transmitter always has an exciton during the application of the pulse. However, when the pulse is cut-off, the number of excitons quickly decay and extinction occurs.

Fig. 7(d) demonstrates that the mean extinction time linearly increases with increasing lifetime of the fluorophores. When the lifetime increases, excitons occupy longer time on the net- work nodes, and the fluorescence rate decreases reciprocally. As a result, the removal rate of the excitons from the network decreases, and excitons survive longer on the network.

3) Channel Capacity: The channel capacity is the maximum mutual information over all input distributions, and it is related to the reliability of the message transmission. Assuming the bit interval satisfies the no-ISI criterion, the capacity of the communication channel between the nanotransmitter and the nanoreceivers is directly related to the successful transmission probability of bit-1. The channel capacity for varying pulse length and mean lifetime of the fluorophores is shown in Fig. 7(e) and Fig. 7(f), and the correlation between the transmission probability of bit-1 presented in Fig. 7(a) and Fig. 7(b) is clearly demonstrated.

4) Achievable Rates: Considering that setting the bit interval as equal to the mean extinction time of excitons, we can neglect the ISI, such that the successive transmissions of messages do not lead to ambiguity in the detection of the messages. Therefore, the achievable rates for varying system parameters are calculated according to (29). Fig. 7(g) and Fig. 7(h) demonstrates the communication rates for different pulse lengths and mean excited state lifetimes. The results reveal that communication can be realized at a rate of $50 \mathrm{kbps}$ over FRET-MAMNET. Note that, the pulse length has almost no effect on the communication rate. Although, increasing pulse length significantly increases the channel reliability, it also linearly increases the extinction time, i.e., bit interval.

\section{CONClusion}

In this paper, based on our recent studies, we introduce FRET-based mobile molecular nanonetworks for the first time in the literature. We present continuous time Markov chain models for FRET-based mobile molecular sensor and actor networks (FRET-MSAN) and FRET-based mobile ad hoc molecular networks (FRET-MAMNET) benefiting from birthdeath processes. We analyze the performance of both nanonetworks in terms of reliable transmission probability, message transmission delay, system throughput, mutual information, information theoretical channel capacity and achievable rates assuming that the network nodes satisfy the rapid-diffusion 


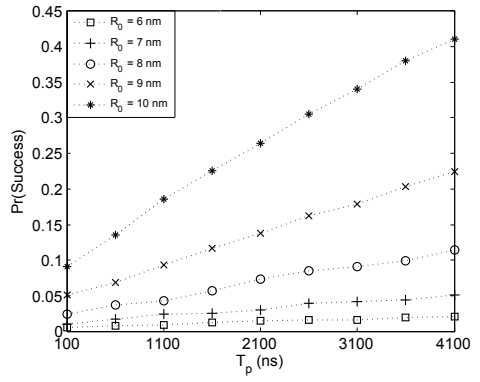

(a) $\operatorname{Pr}$ (success) with varying $T_{p}$ for different $R_{0, h h}=R_{0, h r}=R_{0}$.

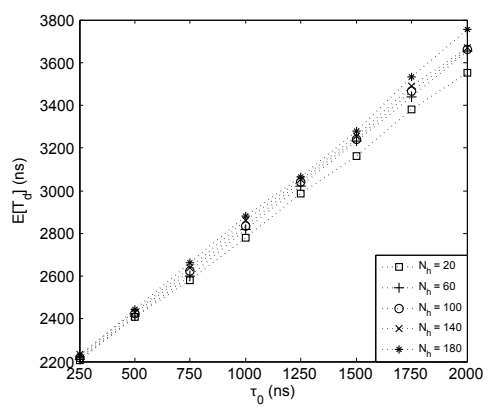

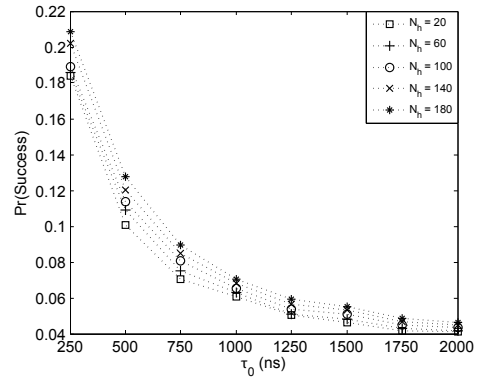

(b) $\operatorname{Pr}($ success $)$ with varying $\tau_{0}$ for different $N_{h}$.

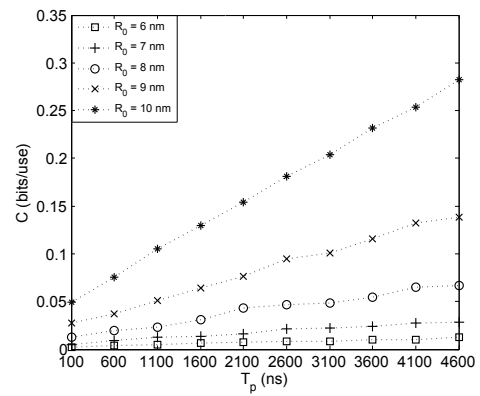

(e) $C$ with varying $T_{p}$ for different $R_{0, h h}=R_{0, h r}=R_{0}$.

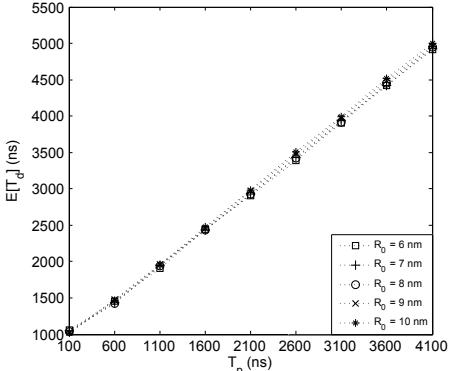

(c) $E\left(T_{d}\right)$ with varying $T_{p}$ for different $R_{0, h h}=R_{0, h r}=R_{0}$.

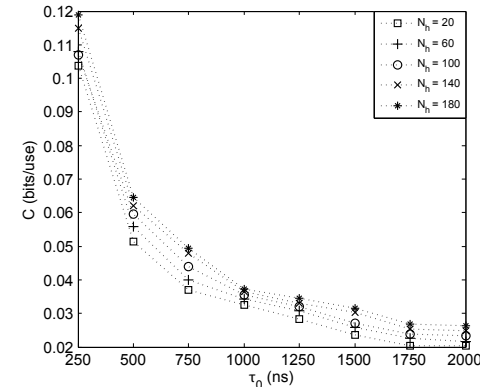

(f) $C$ with varying $\tau_{0}$ for different $N_{h}$.

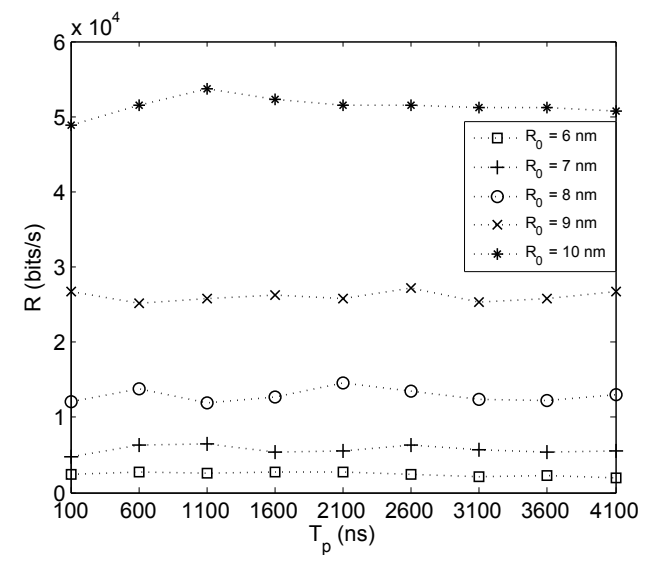

(g) $R$ with varying $T_{p}$ for different $R_{0, h h}=R_{0, h r}=$ $R_{0}$.

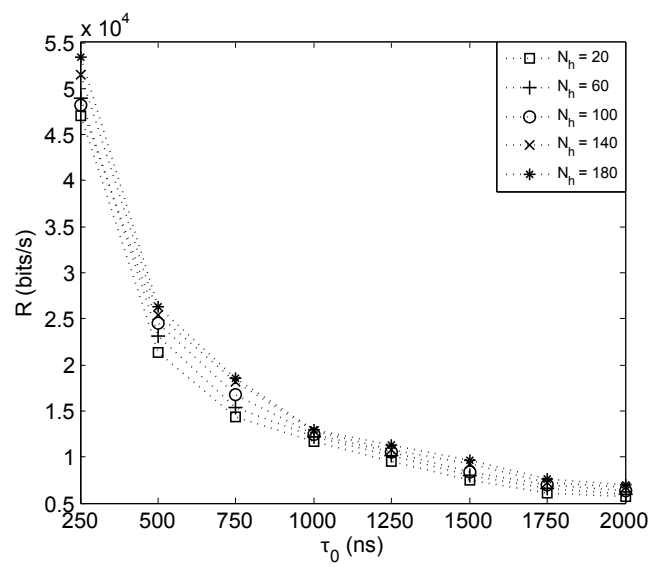

(h) $R$ with varying $\tau_{0}$ for different $N_{h}$.

Fig. 7. The successful detection probability of one-bit message, mean extinction time of excitons, capacity of the channels, and achievable communication rates in FRET-MAMNET.

criterion. The simulation results reveal that it is possible to achieve reliable communication at high-rates with FRET-based mobile molecular networks.

The deployment of bioluminescent sensor fluorophores and photoactive actor fluorophores makes FRET-MSAN promise autonomous applications of target detection and acting at nanoscale such as highly precise PDT applications with singlet oxygen sensitizer fluorophores as actor nodes. FRETMAMNET provides the opportunity of remotely controlling the operation of nanonetworks by an optical information source. This optical link can be made bidirectional if the fluorescence outputs of nanoreceivers are observed by photodetectors, and therefore, it can provide a connection between nanonetworks and larger communication networks, which is currently not possible for other molecular networks proposed so far.

\section{REFERENCES}

[1] I. F. Akyildiz et al., "Nanonetworks: A new communication paradigm," Comput. Netw., vol. 52, no. 12, pp. 2260-2279, 2008.

[2] I. F. Akyildiz and M. J. Jornet, "Electromagnetic wireless nanosensor networks," Nano Commun. Netw., vol. 1, no. 1, pp. 3-19, 2010. 
[3] T. Nakano et al., "Molecular communication for nanomachines using intercellular calcium signaling," in Proc. of the 5th IEEE Conf. on Nanotechnol., vol. 2, pp. 478481, Nagoya, Japan, July 2005.

[4] M. Kuscu, O. B. Akan, "A nanoscale communication channel with fluorescence resonance energy transfer (FRET)," Proc. 1st IEEE Int. Workshop Molecular Nano Scale Commun./IEEE Conf. Comput. Commun. Workshops, Shanghai, China, 2011, pp. 425-430.

[5] J. R. Lakowicz, Principles of fluorescence spectroscopy, 3rd ed., Baltimore, MD: Springer-Verlag, 2006.

[6] T. Förster, "Zwischenmolekulare energiewanderung und fluoreszenz", Annalen der Physik, vol. 437, pp. 55-75, 1948.

[7] L. Stryer, "Fluorescence Energy Transfer as a Spectroscopic Ruler," Annual Review of Biochemistry, vol. 47, pp. 819-846, 1978.

[8] C. Joo, H. Balci, Y. Ishitsuka, C. Buranachai, T. Ha, "Advances in Single-Molecule Fluorescence Methods for Molecular Biology," Ann. Rev. Biochem., vol. 77, pp. 51-76, 2008.

[9] I. L. Medintz, A. R. Clapp, H. Mattoussi, E. R. Goldman, B. Fisher, and J. M. Mauro, "Self-assembled nanoscale biosensors based on quantum dot FRET donors," Nature Materials, vol. 2, pp. 630-638, 2003.

[10] Z. Zhou, M. Yu, H. Yang, K. Huang, F. Li, T. Yi, and C. Huang, "FRET-based sensor for imaging chromium(III) in living cells," Chem. Commun., no. 29, pp. 33873389, 2008.

[11] T. Ritz, A. Damjanovic, K. Schulten, "The quantum physics of photosynthesis," ChemPhysChem, vol. 3, no. 3, pp. 243-248, 2002.

[12] B. E. Hardin, E. T. Hoke, P. B. Armstrong, J. Yum, P. Comte, T. Torres, J. M. J. Frechet, M. K. Nazeeruddin, M. Gratzel, M. D. McGehee, "Increased light harvesting in dye-sensitized solar cells with energy relay dyes," Nature Photonics, vol. 3, pp. 406-411, 2009.

[13] M. Kuscu, O. B. Akan, "A physical channel model and analysis for nanoscale molecular communications with Förster Resonance Energy Transfer (FRET)," IEEE Trans. Nanotechnol., vol. 11, no.1, pp. 200-207, 2012.

[14] M. Kuscu and O. B. Akan, "FRET-Based Nanoscale Point-to-Point and Broadcast Communications with Multi-Exciton Transmission and Channel Routing," IEEE Transactions on Nanobioscience, to be published, 2014.

[15] M. Kuscu, O. B. Akan, "Multi-Step FRET-Based Long-Range Nanoscale Communication Channel," IEEE J. Sel. Areas Commun., vol. 31, no. 12, pp. 715-725, 2013.

[16] D. D. Thomas, W. F. Carlsen, L. Stryer, "Fluorescence Energy Transfer in the Rapid-Diffusion Limit," Proc. Natl. Acad. Sci., vol. 75, no. 12, pp. 5746-5750, 1978.

[17] L. Stryer, "Diffusion-Enhanced Fluorescence Energy Transfer," Ann. Rev. Biophys. Bioeng., vol. 11, pp. 203-222, 1982.

[18] M. Kuscu, O. B. Akan, "FRET-Based Mobile Molecular Nanonetworks," in Proc. IEEE Med-Hoc-Net 2013, Ajaccio, Corsica, France, June 2013.

[19] M. E. J. Newman, "Spread of epidemic disease on networks," Phys. Rev. E, vol. 66, no. 1, pp. 016128(11), 2002.

[20] K. Kinosita, T. Kouyama, A. Ikegami, "Criteria for the Rapid Diffusion Limit of Fluorescence Energy Transfer," J. Chem. Phys., vol. 86, no. 6 , pp. 3273-3282, 1987.

[21] C. H. Contag, M. H. Bachmann, "Advances in in vivo bioluminescence imaging of gene expression," Annu. Rev. Biomed. Eng., vol. 4, pp. 235260, 2002

[22] D. G. Allen, J. R. Blinks, "Calcium transients in aequorin-injected frog cardiac muscle," Nature, vol. 273, no. 5663, pp. 509-513, 1978.

[23] A. C. S. Samia, X. Chen, C. Burda, "Semiconductor quantum dots for photodynamic therapy," J. Am. Chem. Soc., vol. 125, no. 51, pp. 1573615737, 2003.

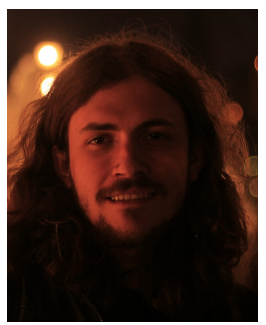

Murat Kuscu [S’11] (mkuscu@ku.edu.tr) received his B.Sc. degree in electrical and electronics engineering from Middle East Technical University, Ankara, Turkey, in July 2011, and his M.Sc. degree in Electrical and Electronics Engineering Department of Koc University, Istanbul, Turkey, in September 2013. He is currently a research assistant at the Nextgeneration and Wireless Communications Laboratory and pursuing his Ph.D. degree in electrical and electronics engineering at Koc University, Istanbul, Turkey. His current research interests include nanoscale and molecular communications.

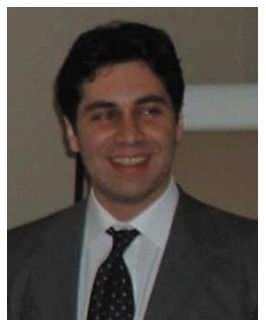

Ozgur B. Akan [M'00-SM'07] (akan@ku.edu.tr) received his Ph.D. degree in electrical and computer engineering from the Broadband and Wireless Networking Laboratory, School of Electrical and Computer Engineering, Georgia Institute of Technology in 2004. He is currently a full professor with the Department of Electrical and Electronics Engineering, Koc University and the director of the Next-generation and Wireless Communications Laboratory. His current research interests are in wireless communications, nano-scale and molecular communications, and information theory. He is an Associate Editor of the IEEE Transactions on Communications, the IEEE Transactions on Vehicular Technology, the International Journal of Communication Systems (Wiley), the Nano Communication Networks Journal (Elsevier), and the European Transactions on Technology. 